\title{
consolidation d'un sol élastoplastique
}

\author{
par \\ J.-P. Magnan et A. Belkeziz \\ Laboratoire Central des Ponts et Chaussées, Paris
}

\section{Introduction}

Depuis les travaux de Sandhu et Wilson (1969), Sandhu (1972, 1976), Christian et Boehmer (1970), Christian et al. (1972), Hwang et al. $(1971,1972)$ et Yokoo et al. (1971 a, b, c), la méthode des éléments finis est devenue un outil courant pour l'analyse (théorique) des problèmes de consolidation. Certains auteurs (Soulié, 1978) ont même trouvé un accord raisonnable entre de "vraies" prévisions du comportement d'un remblai sur sol compressible et les résultats des mesures.

Le Laboratoire Central des Ponts et Chaussées s'est intéressé très tôt à cette technique de calcul numérique et Richard (1975) et Humbert ont introduit dans le programme de calcul par éléments finis ROSALIE un algorithme de traitement des problèmes de consolidation tridimensionnelle dans le cas d'un sol au squelette élastique linéaire (Guellec et al., 1976). ROSALIE a été ultérieurement complété par l'introduction de l'élastoplasticité avec ou sans écrouissage (Belkeziz et Magnan, 1982) dans le module de traitement de la consolidation (ROSALIE-Groupe 9).

L'étude présentée dans cet article a été effectuée en utilisant ce programme. Elle porte sur la consolidation du sol de fondation du remblai $\mathrm{B}$ du site expérimental de remblais sur sols compressibles des Laboratoires des Ponts et Chaussées à Cubzac-les-Ponts (Magnan et al., 1978). La consolidation a été calculée pour différentes hypothèses sur la loi de comportement du squelette du sol:

- élasticité linéaire isotrope,

- élasticité linéaire anisotrope,

- élastoplasticité avec écrouissage (modèle Camclay modifié).

Les résultats des calculs ont été ensuite confrontés aux mesures.

\section{Le programme de calcul : Rosalie-Groupe 9}

Le programme ROSALIE de calcul par éléments finis comprend 16 sous-ensemble appelés * Groupes *, plus trois modules de maillage, et procède suivant le schéma de la figure 1 . Le neuvième groupe, GROUPE 9, traite des problèmes de consolidation et de diffusion de la chaleur. Les éléments utilisés sont du type "déplacements". Avec les notations habituelles pour ce genre de problème :

$e$ - tenseur des déformations (on fait l'hypothèse des petites déformations),

g - vecteur des dérivées premières partielles de la charge hydraulique,

E - tenseur d'élasticité,

$\mathrm{K}$ - tenseur des coefficients de perméabilité,

$\mathrm{H}$ - charge hydraulique, comptée à partir de l'état initial (en supposant $\mathrm{H}=0$, initialement),

$\gamma_{w}$ - poids volumique de l'eau,

$$
\theta=e_{11}+e_{22}+e_{33},
$$

$\dot{\theta}$ - dérivée de $\theta$ par rapport au temps,

$\mathrm{X}^{\top}$ - tenseur transposé du tenseur $\mathrm{X}$,

$F$ - forces volumiques,

$\mathrm{U}$ - déplacements,

$T$ - forces appliquées à la surface du domaine étudié,

$\mathrm{h}$ - débits imposés à la surface du domaine.étudié,

$\Omega$ - domaine étudié,

$S_{\sigma}$ - partie de la surface du domaine $\Omega$ où les contraintes sont imposées,

$S_{v}$ - partie de la surface du domaine $\Omega$ où le débit $h$ est imposé,

la résolution du problème de la consolidation d'un sol élastique linéaire passe par la recherche simultanée d'un champ de déplacement $U$ et d'une distribution de charge hydraulique $H$ qui vérifient :

$$
\begin{array}{r}
\delta \mathcal{F}=\int_{\Omega} e^{\top} E \delta e d \Omega-\int_{\Omega} \gamma_{w} H \delta \theta d \Omega-\int_{\Omega} F^{\top} \delta U d \Omega \\
-\int_{S_{\sigma}} T^{\top} \delta U d S_{\sigma}=0
\end{array}
$$

et

$$
\delta \mathrm{J}=-\int_{\Omega} \gamma_{w} g^{\top} \mathrm{K} \delta \mathrm{g} d \Omega-\int_{\Omega} \dot{\theta} \delta \mathrm{H} d \Omega+\int_{\mathrm{S}_{\mathrm{V}}} \mathrm{h} \delta \mathrm{H} d \mathrm{~S}_{\mathrm{V}}=0
$$




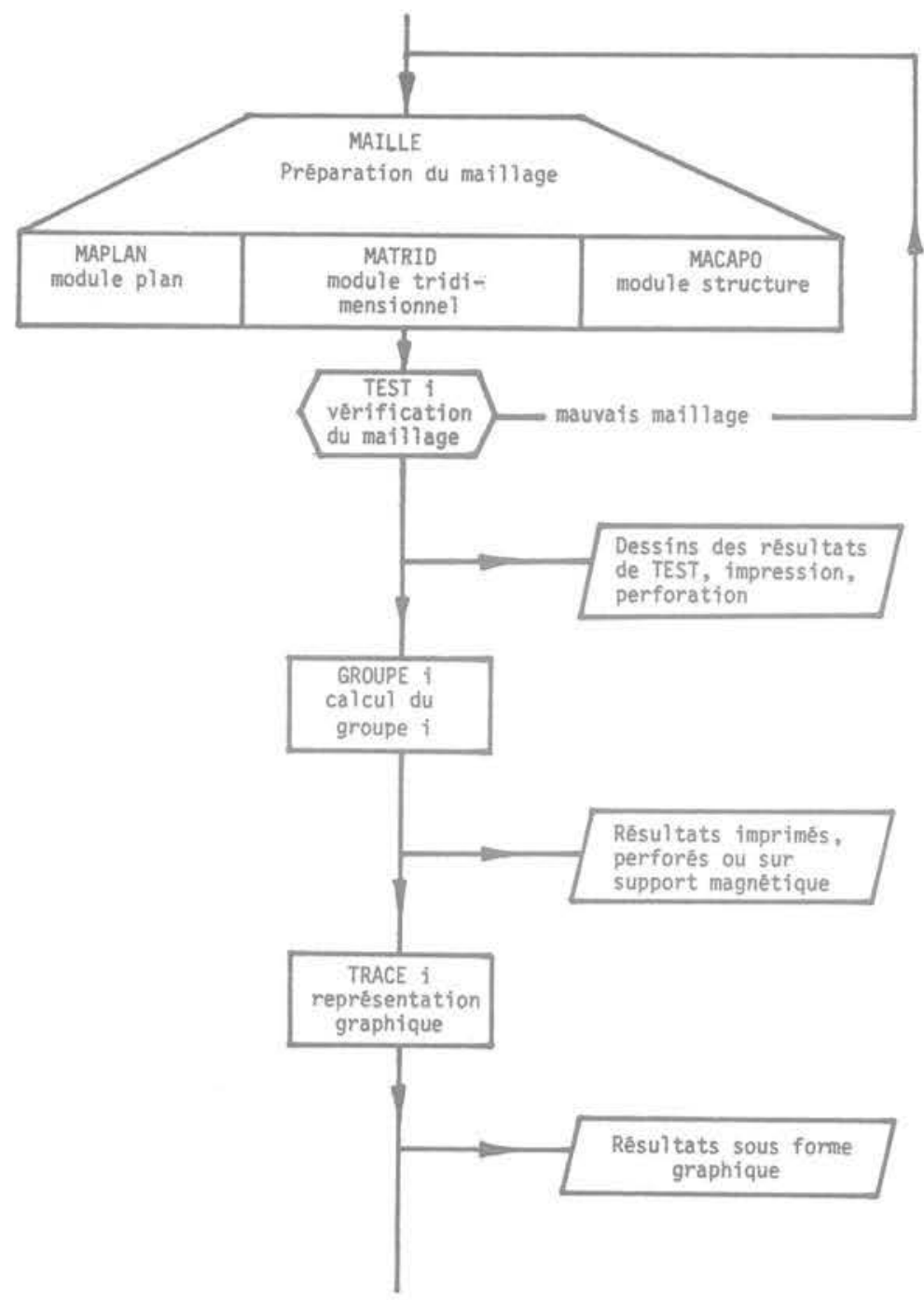

Fig. 1 Structure du programme ROSALIE

pour tout champ de déplacements virtuels admissible (c'est-à-dire vérifiant les conditions de déplacement imposées) $\delta \mathrm{U}$, avec les champs de déformations $\delta \mathrm{e}$ et $\delta \theta$ associés, et pour toute distribution d'incrément de charge $\delta \mathrm{H}$ admissible (c'est-à-dire vérifiant les conditions aux limites imposées à la charge hydraulique $H$ ), avec le champ de dérivées partielles $\delta \mathrm{g}$ associé.

Après discrétisation de l'espace et du temps (par la méthode de Galerkin), l'analyse du problème débouche sur une procédure de résolution itérative sur la base de l'équation matricielle

$$
\mathrm{G}_{\Delta \mathrm{t}} \cdot \mathrm{V}_{\mathrm{t}+\Delta \mathrm{t}}=\mathrm{L}_{\Delta \mathrm{t}}+\mathrm{G}_{\Delta \mathrm{t}}^{*} \cdot \mathrm{V}_{\mathrm{t}}
$$

avec les notations suivantes :

$$
\begin{aligned}
& \begin{array}{ll}
G_{\Delta t}= & R-C \\
-C^{T} & -\frac{2 \Delta t}{3} K
\end{array} \\
& G_{\Delta t}^{*}=\begin{array}{ll}
-\frac{1}{2} R \quad \frac{1}{2} C \\
-C^{\top} \quad \frac{\Delta t}{3} K
\end{array} \\
& L_{\Delta t}=\begin{array}{ll} 
& \frac{1}{2}[F(t)+2 F(t+\Delta t)] \\
& \frac{-\Delta t}{3}[Q(t)+2 Q(t+\Delta t)]
\end{array} \\
& V_{t+\Delta t}=\left[\begin{array}{l}
U(t+\Delta t) \\
H(t+\Delta t)
\end{array}\right], \quad V_{t}=\left[\begin{array}{l}
U(t) \\
H(t)
\end{array}\right],
\end{aligned}
$$

$R$ - matrice de rigidité du squelette du sol, C - matrice de couplage entre l'écoulement et les déformations du squelette,

$\Delta t$ - incrément de temps,

F - matrice-colonne des forces nodales imposées (forces volumiques et forces de surface confondues),

$Q$ - matrice-colonne des débits nodaux imposés.

Cette équation matricielle se résout a facilement ", à la condition d'inverser la matrice $\mathrm{G}_{\Delta t}$, qui est symétrique et tridiagonalisable par blocs. Dans le programme ROSALIE-Groupe 9, l'inversion est réalisée par la méthode directe de Choleski par bande. La résolution donne les déplacements $\mathrm{U}$ et les charges $\mathrm{H}$ au temps $t+\Delta t$, qui permettent de calculer les autres inconnues, et en particulier les contraintes (les conventions de signe du calcul sont celles de la mécanique des milieux continus et non celles de la mécanique des sols).

II faut noter que l'inversion de la matrice $G_{\Delta t}$. qui conditionne pour une grande part le coût total du calcul, doit être effectuée chaque fois que l'on modifie l'incrément de temps $\Delta t$. En pratique, les calculs doivent pour cette raison n'utiliser qu'un nombre limité de valeurs de $\Delta$ t dans chaque cas traité.

L'introduction de l'élastoplasticité dans la procédure de calcul de la consolidation a été effectuée sous forme incrémentale, en supposant que la fonction $F\left(\sigma^{\prime}, k\right)=0$ est à la fois le critère de plasticité et le potentiel plastique (c'est-à-dire qu'on lui applique le principe de normalité), $\sigma^{\prime}$ désignant l'état des contraintes effectives et $\mathrm{k}$ un paramètre d'écrouissage. 
L'analyse du problème de la consolidation dans un sol élastoplastique conduit à l'établissement de deux équations déduites du principe des travaux virtuels et analogues à celles établies dans le cas du squelette élastique linéaire. L'équation (1) relative au squelette du sol est remplacée par l'équation suivante, écrite dans le cas où la plasticité est traitée par la méthode de la contrainte initiale :

$$
\begin{aligned}
& \delta \mathcal{F}=\int_{\Omega} e^{\top} E \delta e d \Omega-\int_{\Omega} \Delta \sigma_{o}(t) \delta e d \Omega-\int_{\Omega} \gamma_{w} H \delta \theta d \Omega \\
&-\int_{\Omega} F^{T} \delta U d \Omega-\int_{S_{\sigma}} T^{\top} \delta U d S_{\sigma} \\
&=0
\end{aligned}
$$

avec les mêmes notations que pour l'équation (1), la correction de contrainte $\Delta \sigma_{0}$ étant obtenue par cumul des corrections introduites à chaque incrément de charge pour caractériser l'état de contrainte réel du sol élastoplastique par rapport à l'état de contrainte fictif calculé en élasticité linéaire :

$$
\begin{aligned}
\Delta \sigma_{0}(t, t+\Delta t) & =\Delta \sigma(t, t+\Delta t)-E d e(t, t+\Delta t), \\
\Delta \sigma_{0} & =\int_{0}^{t} \Delta \sigma_{0}(\tau, \tau+\Delta \tau) d \tau .
\end{aligned}
$$

L'équation relative à l'écoulement est identique à l'équation (2) établie dans le cas du squelette élastique linéaire.

Après discrétisation de l'espace et du temps, on obtient finalement une équation de récurrence de la forme

$$
G_{\Delta t} \cdot V_{\Delta t}^{*}=L_{\Delta t}^{*}+G_{\Delta i}^{* *} \cdot V_{0}^{*}
$$

avec les notations suivantes :

$$
\begin{aligned}
& G_{\Delta t}= R \quad-C \\
&-C^{\top} \quad-\frac{2 \Delta t}{3} K \\
& G_{\Delta t}^{* *}= 0 \frac{1}{2} C \\
& 0 \frac{\Delta t}{3} K \\
& L_{\Delta t}^{*}= \frac{1}{2}\left[F(t)+2 F(t+\Delta t)+2 F_{\Delta \alpha_{0}}(t)\right] \\
&-\frac{\Delta t}{3}[Q(t)+2 Q(t+\Delta t)] \\
& \quad V_{\Delta t}^{*}=\left[\begin{array}{l}
U(t) \\
H(t+\Delta t)
\end{array}\right], \quad V_{0}^{*}=\left[\begin{array}{l}
0 \\
H(t)
\end{array}\right] .
\end{aligned}
$$

$F_{\Delta \sigma_{0}}$ - matrice-colonne des forces nodales dues aux corrections de contraintes,

autres notations, comme pour l'équation (3).

Cette équation qui permet, par un processus itératif, d'obtenir la solution des problèmes de consolidation dans les sols élastoplastiques, appelle deux commentaires :

- d'une part, comme pour l'équation (3), la résolution de l'équation matricielle (4) passe par.l'inversion de la matrice symétrique $\mathrm{G}_{\Delta t}$, qui dépend de l'incrément de temps $\Delta t$ : on a tout intérêt à inverser cette matrice le moins souvent possible, si l'on veut garder le bénéfice du traitement de la plasticité par la méthode des contraintes initiales;

- d'autre part, si l'on regarde la composition de la matrice des inconnues, $V_{\Delta t}^{*}$, on constate qu'elle ne comporte qu'une fonction définie au temps $t+\Delta t$ : la charge hydraulique $H$. Les déplacements $U$ sont définis au temps $t$ et l'on pourrait craindre qu'ils ne restent constants. En réalité, ils varient, par l'intermédiaire des corrections de contraintes qu'un processus itératif annexe, inclus dans le processus itératif général relatif au temps, cumule jusqu'à ramener en tout point du maillage l'état des contraintes effectives sur le critère de plasticité.

La figure 2 explique le déroulement des calculs de consolidation dans le cas des sols élastoplastiques.

Dans son état actuel, le programme ROSALIEGroupe 9 permet de traiter la consolidation de sols dont le critère de plasticité du squelette est l'un des critères de Tresca, Von Misès ou Mohr-Coulomb ou, depuis les travaux de Dang et Magnan (1977), le critère d'élastoplasticité avec écrouissage du modèle Camclay modifié.

\section{Le cas traité : remblai B du site expérimental de Cubzac-les-Ponts}

L'étude a été réalisée sur le cas d'un remblai réel, édifié en 1975 par les Laboratoires des Ponts et Chaussées sur la rive nord de la Dordogne, à Cubzac-les-Ponts (Gironde). Ce remblai a fait l'objet de nombreuses études théoriques et expérimentales et a été décrit dans plusieurs publications antérieures (Dang et Magnan, 1977; Magnan et al., 1978; Shahanguian, 1980; Baghery, 1980). Nous nous contenterons de rappeler ici la géométrie du remblai et du sol de fondation (fig. 3) et de reproduire la coupe géotechnique du sol de fondation, telle qu'elle résulte de la reconnaissance effectuée avant la construction du remblai (Tableau I).

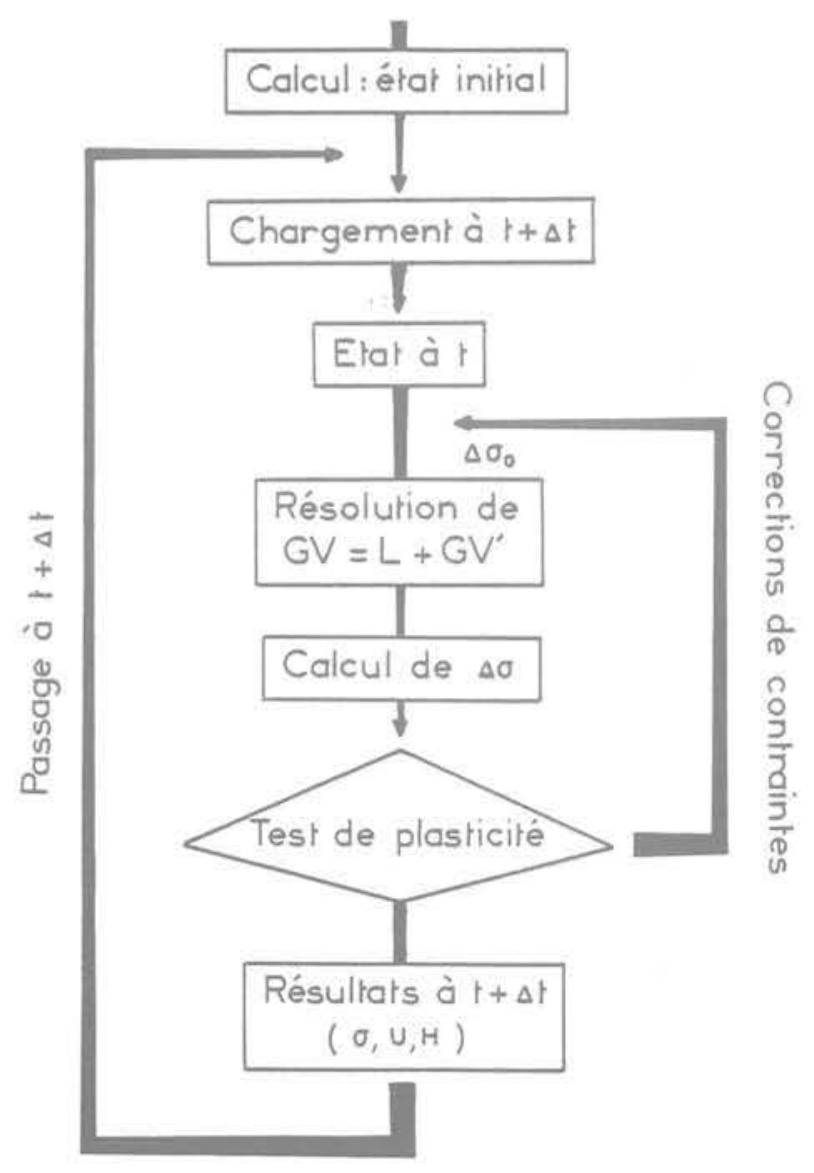

Fig. 2 
Tableau 1

Caractéristiques de compressibilité des sols de fondation du remblai B du site expérimental de Cubzac-les-Ponts

\begin{tabular}{|c|c|c|c|c|c|c|c|c|c|c|c|c|c|c|c|c|c|}
\hline \multirow{3}{*}{ Couches } & \multirow{3}{*}{$\theta_{0}$} & \multirow[b]{3}{*}{$\begin{array}{c}\sigma_{v 0} \\
(\mathrm{kPa})\end{array}$} & \multirow[b]{3}{*}{$\begin{array}{c}\sigma_{\dot{p}}^{\prime} \\
(\mathrm{kPa})\end{array}$} & \multirow{3}{*}{$\mathrm{C}_{\mathrm{m}}$} & \multirow{3}{*}{$\mathrm{c}_{\mathrm{c}}$} & \multirow{3}{*}{ A } & \multirow{3}{*}{8} & \multicolumn{6}{|c|}{ Drainage vertical } & \multicolumn{4}{|c|}{ Drainage horizontal } \\
\hline & & & & & & & & \multicolumn{2}{|c|}{$\sigma^{\prime}=\alpha_{\text {so }}$} & \multicolumn{2}{|c|}{$\sigma^{\prime}=\sigma_{00}+\frac{48,3}{2}$} & \multicolumn{2}{|c|}{$\sigma^{\prime}=\sigma_{\mathrm{vo}}^{\prime}+48.3$} & \multirow{2}{*}{$A_{n}$} & \multirow{2}{*}{$\theta_{n}$} & $\sigma^{\prime}=\sigma_{v 0}$ & \multirow{2}{*}{$\begin{array}{l}\text { pour } \\
\sigma^{\prime}=\sigma_{v o} \\
k_{n} / k_{v}\end{array}$} \\
\hline & & & & & & & & $\begin{array}{c}\mathrm{k}_{\mathrm{v}} \\
(\mathrm{cm} / \mathrm{s})\end{array}$ & $\begin{array}{c}\mathrm{c}_{\mathrm{v}} \\
\left(\mathrm{cm}^{2} / \mathrm{s}\right)\end{array}$ & $\begin{array}{c}k_{v} \\
(\mathrm{~cm} / \mathrm{s})\end{array}$ & $\begin{array}{c}c_{v} \\
\left(\mathrm{~cm}^{2} / \mathrm{s}\right)\end{array}$ & $\begin{array}{c}k_{v} \\
(\mathrm{~cm} / \mathrm{s})\end{array}$ & $\begin{array}{c}c_{v} \\
\left(\mathrm{~cm}^{2} / \mathrm{s}\right)\end{array}$ & & & $\begin{array}{c}k_{h} \\
(\mathrm{~cm} / \mathrm{s})\end{array}$ & \\
\hline 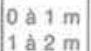 & $\begin{array}{l}1 \\
2,6\end{array}$ & $\begin{array}{l}8.4 \\
20\end{array}$ & $\begin{array}{l}78 \\
68\end{array}$ & $\begin{array}{l}0,04 \\
0,05\end{array}$ & $\begin{array}{l}0,28 \\
1,22\end{array}$ & $\begin{array}{l}2,2 \\
8.24\end{array}$ & $\mid \begin{array}{l}0.17 \\
0.81\end{array}$ & $\begin{array}{r}9 \cdot 10^{-8} \\
125 \cdot 10^{-7}\end{array}$ & $\begin{array}{l}8,6 \cdot 10^{-4} \\
42 \cdot 10^{-3}\end{array}$ & $\begin{array}{l}6.5 \cdot 10^{-8} \\
12 \cdot 10^{-7}\end{array}$ & $\begin{array}{l}2,4 \cdot 10^{-3} \\
87 \cdot 10^{-a}\end{array}$ & $\begin{array}{l}5,7 \cdot 10^{-8} \\
115 \cdot 10^{-7}\end{array}$ & $3.6 \cdot 10^{-3}$ & 2.3 & 0.19 & $4.6 \cdot 10^{-8}$ & 0,51 \\
\hline $2 \mathrm{a} 3 \mathrm{~m}$ & 3,15 & 24 & 36 & 0,21 & 1.75 & 13.4 & 1.39 & $4.3 \cdot 10^{-8}$ & $4,7 \cdot 10^{-4}$ & $\begin{array}{l}1.2 \cdot 10^{-1} \\
2.5 \cdot 10^{-8}\end{array}$ & $\begin{array}{l}8,7 \cdot 10^{-3} \\
6,6 \cdot 10^{-5}\end{array}$ & $\begin{array}{r}1,15 \cdot 10^{-7} \\
1,7 \cdot 10^{-8}\end{array}$ & $\begin{array}{l}1,3 \cdot 10^{-2} \\
5,7 \cdot 10^{-5}\end{array}$ & 11 & 1,13 & $1,4 \cdot 10^{-7}$ & 3.26 \\
\hline 3 a $4 \mathrm{~m}$ & 3,3 & 28 & 36 & 0,18 & 1,70 & 10,5 & 1,03 & $1 \cdot 10^{-7}$ & $1,6 \cdot 10^{-3}$ & $5.4 \cdot 10^{-8}$ & $1,5 \cdot 10^{-4}$ & $2,9 \cdot 10^{-8}$ & $1,1 \cdot 10^{-4}$ & 12 & 1,24 & $3,6 \cdot 10^{-7}$ & $\begin{array}{l}3,20 \\
3,60\end{array}$ \\
\hline 4 à $5 \mathrm{~m}$ & 2,42 & 32 & 38,5 & 0,12 & 1,28 & 8.95 & 0.93 & $9.7 \cdot 10^{-8}$ & $2 \cdot 10^{-3}$ & $5.6 \cdot 10^{-6}$ & $1,8 \cdot 10^{-4}$ & $3,4 \cdot 10^{-6}$ & $1,5 \cdot 10^{-4}$ & 10 & 1.07 & $1 \cdot 10^{-7}$ & 3,09 \\
\hline 5 à $6 m$ & 2,07 & 36 & 41 & 0,10 & 1,18 & 7,46 & 0.78 & $1.2 \cdot 10^{-7}$ & $3.2 \cdot 10^{-3}$ & $6.8 \cdot 10^{-8}$ & $2,3 \cdot 10^{-4}$ & $4,1 \cdot 10^{-8}$ & $1.8 \cdot 10^{-4}$ & 6.23 & 0.65 & $1.9 \cdot 10^{-7}$ & 2,42 \\
\hline 6 a $7 \mathrm{~m}$ & 2,13 & 40 & 46 & 0,08 & 1.11 & 7.01 & 0,70 & $1,1 \cdot 10^{-7}$ & $3.9 \cdot 10^{-3}$ & $6.4 \cdot 10^{-8}$ & $2.5 \cdot 10^{-4}$ & $3,9 \cdot 10^{-6}$ & $2 \cdot 10^{-4}$ & 8 & 0.84 & $1,1 \cdot 10^{-7}$ & 1 \\
\hline 7 aे $8 \mathrm{~m}$ & 2,5 & 45 & 57 & 0,10 & 1,30 & 8,42 & 0,84 & $9 \cdot 10^{-6}$ & $3,3 \cdot 10^{-3}$ & $6.6 \cdot 10^{-8}$ & $2,7 \cdot 10^{-4}$ & $4,1 \cdot 10^{-8}$ & $2.2 \cdot 10^{-4}$ & 7,4 & 0.74 & $1,5 \cdot 10^{-7}$ & 1.67 \\
\hline 8 à $9 \mathrm{~m}$ & 2,2 & 48 & 68 & 0,11 & 1,20 & 7.52 & 0.75 & $8.2 \cdot 10^{-8}$ & $2,6 \cdot 10^{-3}$ & $7 \cdot 10^{-8}$ & $3 \cdot 10^{-4}$ & $4,4 \cdot 10^{-8}$ & $2.4 \cdot 10^{-4}$ & & & & \\
\hline
\end{tabular}

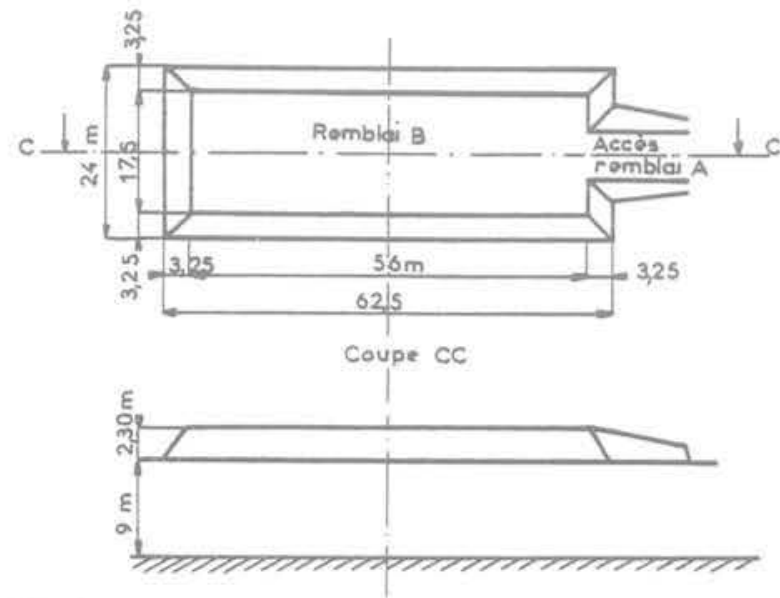

Fig. 3

\subsection{Liste et hypothèses des calculs effectués}

Trois séries de calculs ont été réalisées sur le remblai B de Cubzac-les-Ponts :

- deux calculs de consolidation dans l'hypothèse d'un sol au squelette élastique isotrope (sol de fondation homogène et sol de fondation divisé en cinq couches horizontales);

- deux calculs de consolidation dans l'hypothèse d'un sol au squelette élastique anisotrope ou plus précisément orthotrope à symétrie de révolution (sol de fondation homogène et sol de fondation divisé en cinq couches horizontales);

- un calcul de consolidation dans l'hypothèse oủ le comportement élastoplastique du sol est régi par le modèle Cam-clay modifié (sol de fondation divisé en cinq couches horizontales).

Dans tous les cas, le remblai a été remplacé par une distribution trapézoïdale de pressions à la surface du massif de sol.

\section{Maillages et discrétisation du temps}

Les calculs ont tous été effectués sur deux maillages correspondant respectivement au cas du massif de fondation homogène et au cas du massif de fondation divisé en cinq couches (fig. 4). Ces maillages sont composés d'éléments rectangulaires à huit nœuds, à l'intérieur desquels les fonctions d'interpolation des déplacements et de la charge hydraulique sont des polynômes du second degré.

\section{Massif homogène}

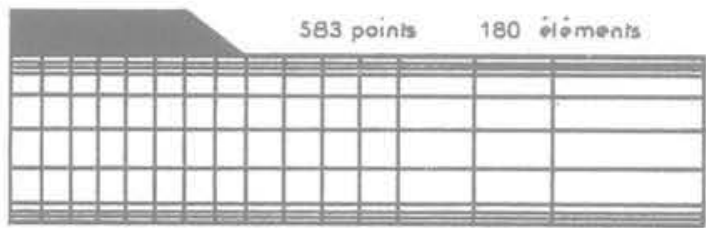

Massif hétérogène

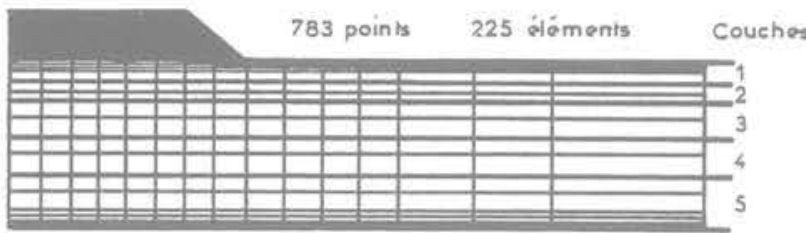

Fig. 4

Pour la discrétisation du temps, on a cherché simultanément à :

- représenter aussi fidèlement que possible la vitesse de construction du remblai,

- couvrir une période de temps suffisamment étendue (20 ans),

- limiter au strict minimum les modifications de l'incrément de temps $\Delta t$ au cours du calcul, pour limiter le nombre d'inversions de la matrice $\mathrm{G}_{\Delta \mathrm{t}}$.

Ceci a conduit au choix des incréments de temps suivants (fig. 5 ) :

- six incréments de 1 jour.

- suivis de quatre incréments de 10 jours,

- suivis de dix incréments de 100 jours,

- suivis de cinq incréments de 1000 jours.

L'utilisation de quatre valeurs de $\Delta t$ permet de n'avoir que quatre inversions de la matrice $G_{\Delta t}$ à effectuer.

Propriétés du sol (élasticité linéaire isotrope)

Chaque couche de sol est caractérisée par cinq paramètres :

- le coefficient de pression des terres au repos, $\mathrm{K}_{\mathrm{o}}$.

- le module d'Young E et le coefficient de Poisson $v$,

- les coefficients de perméabilité verticale $k_{v}$ et horizontale $\mathrm{k}_{\mathrm{h}}$.

Faute de résultats expérimentaux, on a attribué au coefficient de Poisson la valeur arbitraire $v=0,35$ et au coefficient $K_{0}$ la valeur également arbitraire $K_{0}=0,43$. 


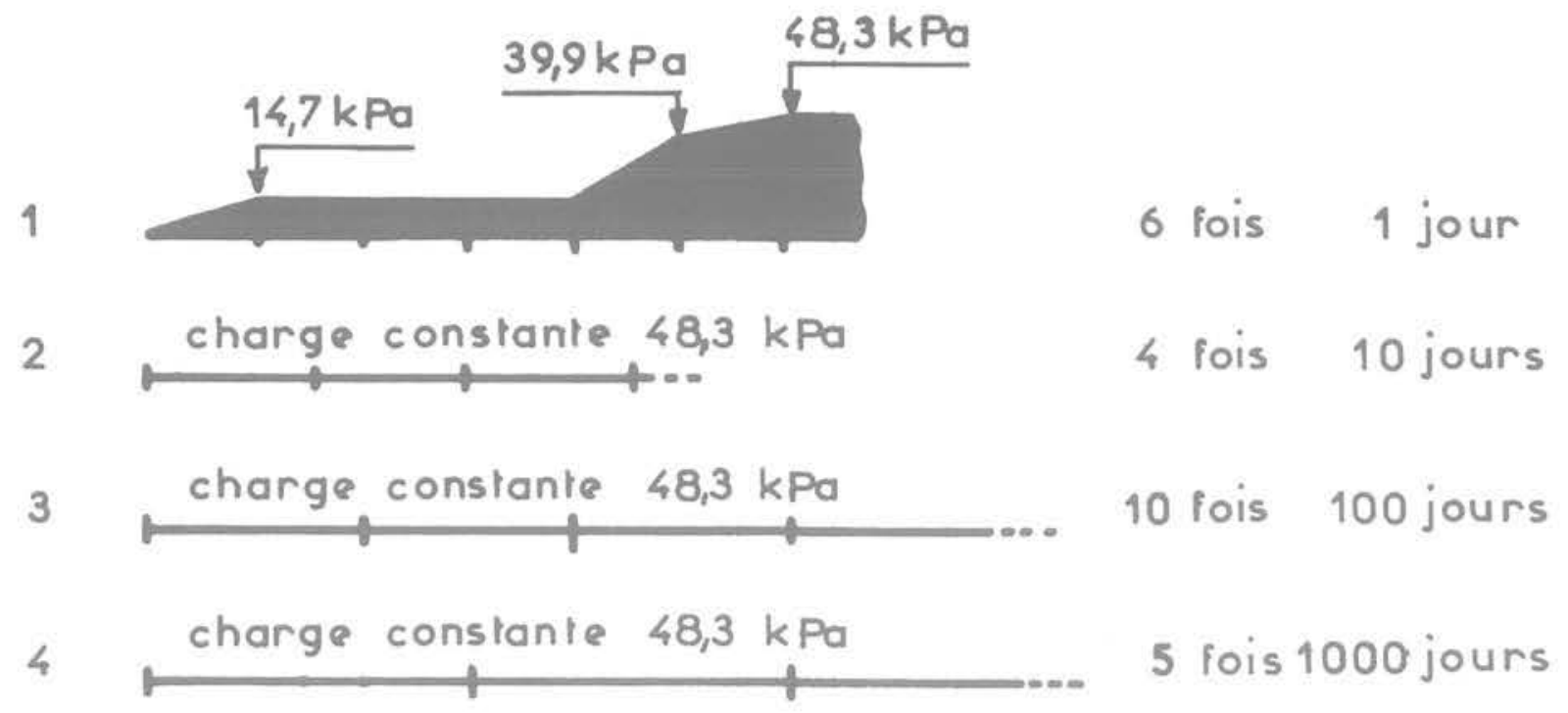

Fig. 5

Tableau 2

Hypothèses du calcul (sol homogène élastique isotrope)

\begin{tabular}{|c|c|c|c|c|c|}
\hline Couche & $v$ & $\mathrm{E}(\mathrm{kPa})$ & $\mathrm{k}_{\mathrm{h}}(\mathrm{m} / \mathrm{s})$ & $\mathrm{k}_{\mathrm{v}}(\mathrm{m} / \mathrm{s})$ & $\mathrm{K}_{\mathrm{o}}$ \\
\hline $0 \mathrm{~m}-9 \mathrm{~m}$ & 0,35 & 300 & $1,62 \cdot 10^{-9}$ & $0,96 \cdot 10^{-9}$ & 0,43 \\
\hline
\end{tabular}

Tableau 3

Hypothèses du calcul (sol hétérogène élastique isotrope)

\begin{tabular}{|c|c|c|c|c|c|}
\hline Couche & $v$ & $\mathrm{E}(\mathrm{kPa})$ & $\mathrm{k}_{\mathrm{h}}(\mathrm{m} / \mathrm{s})$ & $\mathrm{k}_{\mathrm{v}}(\mathrm{m} / \mathrm{s})$ & $\mathrm{K}_{\mathrm{o}}$ \\
\hline $0 \mathrm{~m}-1 \mathrm{~m}$ & 0,35 & 1814 & $0,46 \cdot 10^{-9}$ & $0,90 \cdot 10^{-9}$ & 0,43 \\
$1 \mathrm{~m}-2 \mathrm{~m}$ & 0,35 & 3748 & $1,25 \cdot 10^{-9}$ & $1,25 \cdot 10^{-9}$ & 0,43 \\
$2 \mathrm{~m}-4 \mathrm{~m}$ & 0,35 & 222 & $2,50 \cdot 10^{-9}$ & $0,72 \cdot 10^{-9}$ & 0,43 \\
$4 \mathrm{~m}-6 \mathrm{~m}$ & 0,35 & 246 & $2,95 \cdot 10^{-9}$ & $1,08 \cdot 10^{-9}$ & 0,43 \\
$6 \mathrm{~m}-9 \mathrm{~m}$ & 0,35 & 382 & $1,30 \cdot 10^{-9}$ & $0,94 \cdot 10^{-9}$ & 0,43 \\
\hline
\end{tabular}

Le module d'Young a été déduit du module œdométrique $\mathrm{E}_{\mathrm{cod}}$ moyen entre l'état initial du sol et son état final sous le poids du remblai, en utilisant la valeur choisie pour $v$. Les coefficients de perméabilité du sol ont été choisis d'après les valeurs indiquées dans la coupe géotechnique (tableau 1).

Les tableaux 2 et 3 rassemblent les valeurs des paramètres adoptées pour les calculs dans les cas du sol homogène et du sol hétérogène.

Propriétés du sol (élasticité linéaire anisotrope)

Chaque couche de sol est caractérisée par huit paramètres :

- le coefficient de pression des terres au repos, $\mathrm{K}_{0}$,

- les cinq paramètres de l'élasticité linéaire orthotrope à symétrie de révolution: $E_{v}, E_{h}, \nu_{h}, \nu_{v}$ et $G_{v}$,

- les coefficients de perméabilité verticale $k_{v}$ et horizontale $k_{h}$.

Les valeurs du coefficient $K_{0}$ et des coefficients de perméabilité $k_{v}$ et $k_{v}$ sont identiques à celles adoptées pour le calcul en élasticité isotrope. Pour les paramètres d'élasticité, pour lesquels on ne disposait pas de valeurs expérimentales, on a fait les choix suivants :

- le module d'élasticité verticale $E_{\mathrm{v}}$ est égal au module d'Young de l'élasticité isotrope,

- le rapport $E_{n} / E_{v}$ est égal à 2 , valeur médiane de l'intervalle de variation indiqué pour les argiles dans la synthèse bibliographique de Garnier (1973),

- le rapport $G_{v} / E_{v}$ est égal a 0,5 , d'après une synthèse des publications concernant ce sujet,

- les valeurs de $\nu_{\mathrm{h}}$ et $\nu_{x}$ ont été choisies après une étude paramétrique, de façon à assurer que les déplacements horizontaux continuent vers l'extérieur après la fin du chargement, que le rapport du déplacement latéral maximal au tassement sous l'axe du remblai reste de l'ordre de 0,2 et que le rapport du tassement final au tassement initial soit voisin de 10.

Les tableaux 4 et 5 présentent les valeurs des paramètres utilisées pour les calculs.

Propriétés du sol (élastoplasticité avec écrouissage) 
Tableau 4

Hypothèses du calcul (élasticité linéaire anisotrope, sol homogène)

\begin{tabular}{|c|c|c|c|c|c|c|c|c|}
\hline Couche & $E_{h}(\mathrm{kPa})$ & $\mathrm{E}_{\mathrm{v}}(\mathrm{kPa})$ & $\mathrm{G}_{\mathrm{v}}(\mathrm{kPa})$ & $\nu \mathrm{h}$ & $\nu \mathrm{v}$ & $\mathrm{k}_{\mathrm{h}}(\mathrm{m} / \mathrm{s})$ & $\mathrm{k}_{\mathrm{v}}(\mathrm{m} / \mathrm{s})$ & $\mathrm{K}_{\mathrm{o}}$ \\
\hline $0 \mathrm{~m}-9 \mathrm{~m}$ & 600 & 300 & 150 & 0,9 & 0,1 & $1,62 \cdot 10^{-9}$ & $0,96 \cdot 10^{-9}$ & 0,43 \\
\hline
\end{tabular}

Tableau 5

Hypothèses du calcul (élasticité linéaire anisotrope, sol hétérogène)

\begin{tabular}{|c|c|c|c|c|c|c|c|c|}
\hline Couche & $E_{n}(\mathrm{kPa})$ & $\mathrm{E}_{\mathrm{v}}(\mathrm{kPa})$ & $\mathrm{G}_{\mathrm{v}}(\mathrm{kPa})$ & $\nu \mathrm{h}$ & $\nu \mathrm{v}$ & $\mathrm{K}_{\mathrm{h}}(\mathrm{m} / \mathrm{s})$ & $\mathrm{k}_{\mathrm{v}}(\mathrm{m} / \mathrm{s})$ & $\mathrm{K}_{0}$ \\
\hline $0 \mathrm{~m}-1 \mathrm{~m}$ & 3628 & 1814 & 907 & 0,8 & 0,1 & $1,80 \cdot 10^{-9}$ & $1,80 \cdot 10^{-9}$ & 0,43 \\
$1 \mathrm{~m}-2 \mathrm{~m}$ & 7496 & 3748 & 1874 & 0,8 & 0,1 & $1,25 \cdot 10^{-9}$ & $1,21 \cdot 10^{-9}$ & 0,43 \\
$2 \mathrm{~m}-4 \mathrm{~m}$ & 445 & 222 & 111 & 0,875 & 0,1 & $2,50 \cdot 10^{-9}$ & $0,72 \cdot 10^{-9}$ & 0,43 \\
$4 \mathrm{~m}-6 \mathrm{~m}$ & 492 & 246 & 123 & 0,875 & 0,1 & $2,95 \cdot 10^{-9}$ & $1,08 \cdot 10^{-9}$ & 0,43 \\
$6 \mathrm{~m}-9 \mathrm{~m}$ & 764 & 382 & 191 & 0,850 & 0,1 & $2,95 \cdot 10^{-9}$ & $1,88 \cdot 10^{-9}$ & 0,43 \\
\hline
\end{tabular}

Tableau 6

Hypothèses du calcul (élastoplasticité avec écrouissage)

\begin{tabular}{|c|c|c|c|c|c|c|c|c|c|c|}
\hline \multirow{2}{*}{ Couches } & \multicolumn{3}{|c|}{$\begin{array}{c}\text { Paramètres } \\
\text { d'élasticité }\end{array}$} & \multicolumn{6}{c|}{ Paramètres de plasticité } & \multicolumn{4}{c|}{ Ecoulement } \\
\cline { 2 - 12 } & $\mathrm{E}(\mathrm{kPa})$ & $v$ & $\lambda$ & $x$ & $\mathrm{M}$ & $e_{0}$ & $e_{\lambda_{0}}$ & $e_{0}^{p}$ & $\mathrm{k}_{\mathrm{h}}(\mathrm{m} / \mathrm{s})$ & $\mathrm{k}_{\mathrm{v}}(\mathrm{m} / \mathrm{s})$ \\
\hline $0 \mathrm{~m}-1 \mathrm{~m}$ & 6000 & 0,35 & 0,12 & 0,017 & 1,2 & 1,00 & 1,47 & 1,03 & $1,80 \cdot 10^{-9}$ & $1,80 \cdot 10^{-9}$ \\
$1 \mathrm{~m}-2 \mathrm{~m}$ & 7600 & 0,35 & 0,53 & 0,022 & 1,2 & 2,60 & 4,72 & 2,66 & $1,25 \cdot 10^{-9}$ & $1,21 \cdot 10^{-9}$ \\
$2 \mathrm{~m}-4 \mathrm{~m}$ & 1220 & 0,35 & 0,75 & 0,085 & 1,2 & 3,23 & 5,78 & 3,48 & $2,50 \cdot 10^{-9}$ & $0,72 \cdot 10^{-9}$ \\
$4 \mathrm{~m}-6 \mathrm{~m}$ & 1830 & 0,35 & 0,53 & 0,048 & 1,2 & 2,25 & 4,11 & 2,34 & $2,95 \cdot 10^{-9}$ & $1,08 \cdot 10^{-9}$ \\
$6 \mathrm{~m}-9 \mathrm{~m}$ & 2990 & 0,35 & 0,52 & 0,042 & 1,2 & 2,28 & 4,29 & 2,24 & $2,95 \cdot 10^{-9}$ & $1,88 \cdot 10^{-9}$ \\
\hline
\end{tabular}

Chaque couche de sol est caractérisée par onze paramètres :

- le coefficient de pression des terres au repos, $\mathrm{K}_{0}$.

- les huit paramètres qui décrivent la surface d'état limite du modèle Cam-clay modifié $\left(\lambda, x, M, e_{\lambda_{0}}, e_{0}^{P}\right)$, le comportement élastique isotrope du squelette à l'intérieur de la surface d'état limite $(E, v)$ et l'état initial du sol $\left(e_{0}\right)$,

- les deux coefficients de perméabilité verticale $k_{v}$ et horizontale $\mathrm{k}_{\mathrm{h}}$.

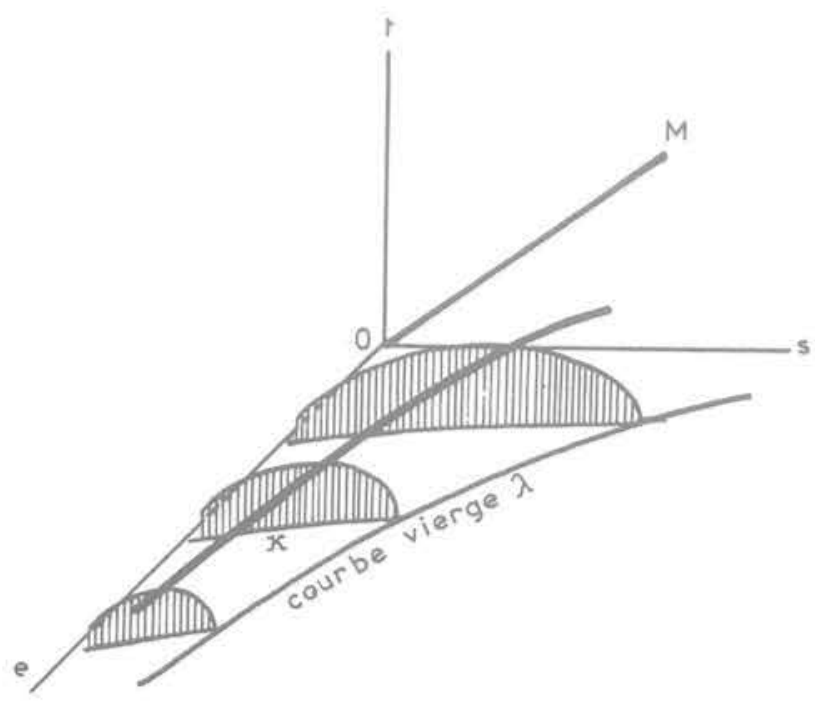

Fig. 6
Pour ce calcul, on a pris un coefficient de pression des terres au repos $K_{0}=0,5$. Les coefficients de perméabilité sont identiques à ceux des calculs précédents. La valeur du coefficient de Poisson $v$ a été fixée cette fois encore à 0,35 . Le module d'Young $\mathrm{E}$ a été obtenu par linéarisation des courbes de déchargementrechargement isotrope (courbe de pente $x$ sur la figure 6).

La valeur des paramètres de la courbe d'état limite a été déduite des résultats des essais œdométriques, sauf pour $M$, calculé à l'aide de la formule de Burland

$$
M=\frac{6 \sin \phi^{\prime}}{3-\sin \phi^{\prime}}
$$

( $\phi^{\prime}$ - angle de frottement interne du squelette du sol).

Le tableau 6 présente les valeurs des paramètres utilisées pour les calculs.

\subsection{Résultats des calculs}

Squelette élastique isotrope

La figure 7 regroupe les résultats des deux calculs effectués avec un massif de sol homogène et un massif de sol hétérogène.

On observe sur cette figure que le tassement initial de la surface du sol sous l'axe du remblai est plus important dans le cas du sol homogène, mais qu'ultérieurement le tassement se poursuit à la même vitesse dans les deux cas. 

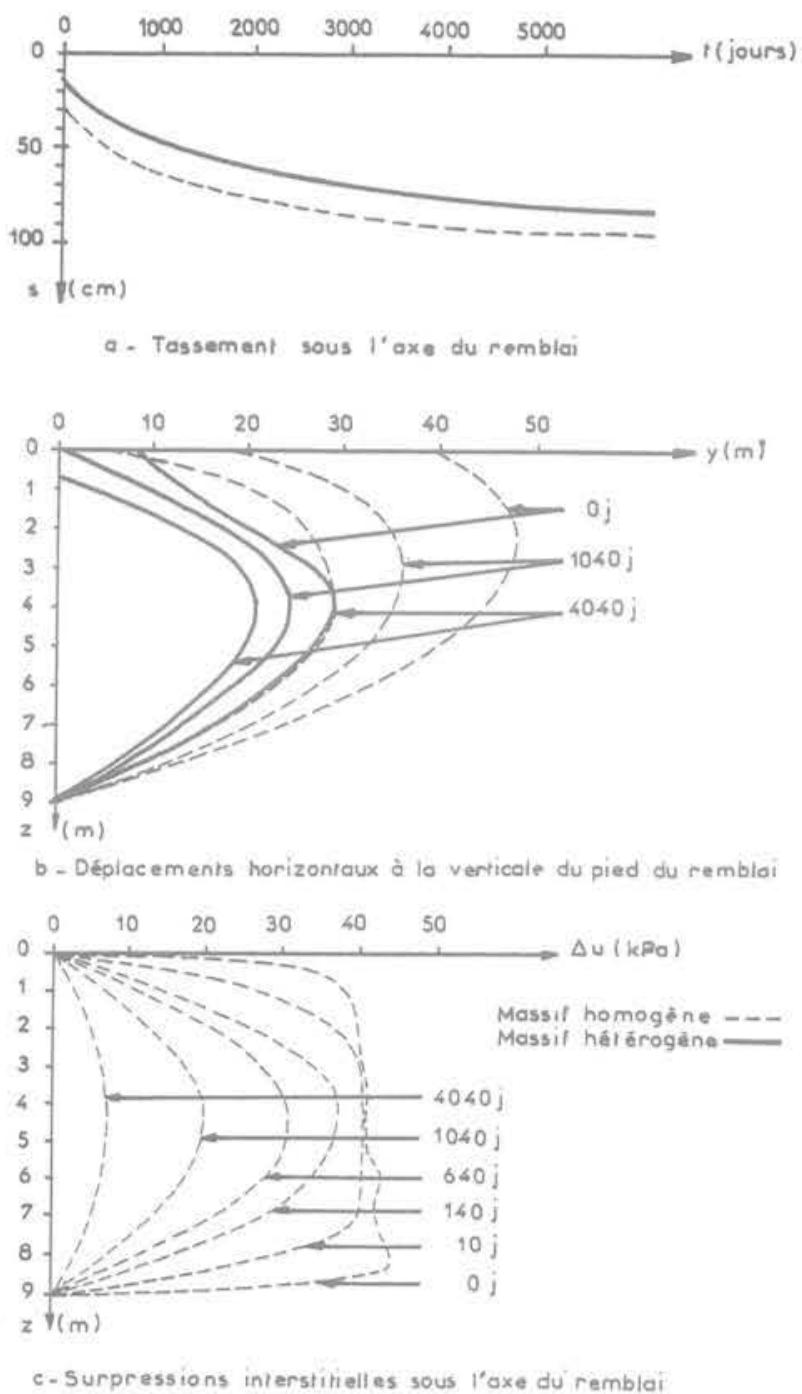

Fig. 7

Le déplacement horizontal du sol à la verticale du pied du remblai est beaucoup plus important dans le cas du massif de sol homogène, ce qui s'explique par la rigidité plus grande des deux couches de surface dans le cas du massif hétérogène. Comme dans tous les calculs de consolidation des sols élastiques isotropes, on obtient une déformée latérale qui revient vers l'intérieur du remblai au cours de la consolidation, ce qui n'est jamais observé sur les remblais réels.

Les isochrones de surpression interstitielle sous l'axe du remblai sont peu différentes lorsque l'on passe du sol homogène au sol hétérogène. On n'a, pour cette raison, représenté qu'un seul réseau d'isochrones sur la figure 7. On note sur l'isochrone correspondant à la fin du chargement $(\mathrm{t}=\mathrm{O} \mathrm{j})$ des ondulations provoquées par les oscillations de la solution numérique lors des premières itérations du calcul.

\section{Squelette élastique anisotrope}

La figure 8 regroupe les résultats des deux calculs effectués dans l'hypothèse du squelette élastique anisotrope (sol homogène et sol hétérogène).

Le tassement du milieu du remblai est le même dans les deux cas.

Les déplacements horizontaux sont plus importants dans le cas du sol homogène, toujours parce que les couches supérieures du sol hétérogène sont plus rigides. Les résultats des calculs sont conformes à ce

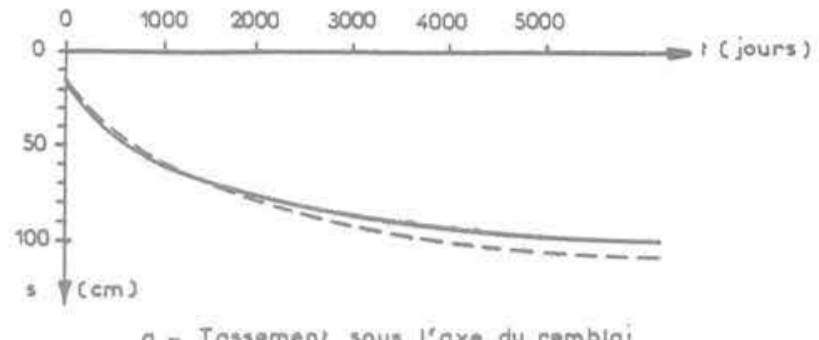

$\begin{array}{llllllllllll}0 & 2 & 6 & 8 & 10 & 12 & 14 & 16 & 18 & 20 & 22\end{array}$
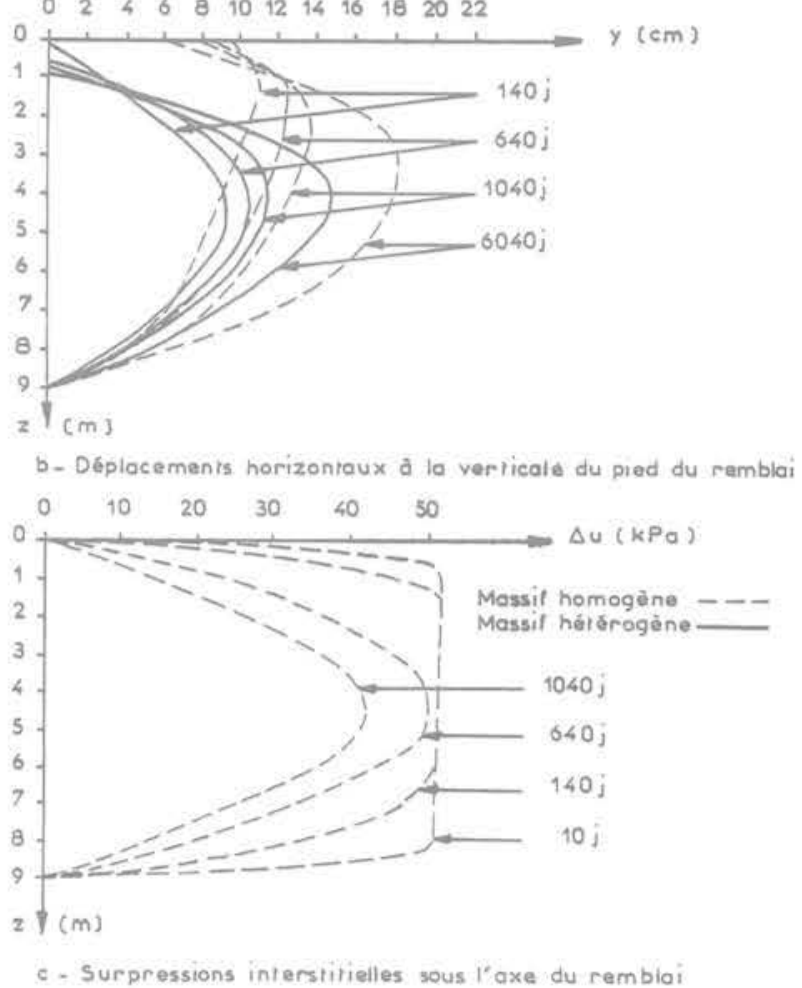

Fig. 8

que l'on attendait, compte tenu de la façon dont on a choisi la valeur des paramètres $\nu_{v}$ et $\nu_{n}$ : les déplacements horizontaux augmentent au cours de la consolidation.

Cette fois encore les isochrones de surpression interstitielle diffèrent peu d'un calcul à l'autre et ce sont les isochrones pour le sol homogène qui sont représentées sur la figure 8 .

\section{Squelette élastoplastique avec écrouissage}

L'évolution des zones plastiques (au sens du modèle Cam-clay modifié) au cours de la construction du remblai puis de la consolidation est représentée sur la figure 9 . On note que le sol devient progressivement plastique en commençant par la partie inférieure de la couche. Initialement, l'état limite est atteint dans la partie de la surface d'état limite où les déformations plastiques entraînent un écrouissage du sol. Au cours de la consolidation (pour $t=1040 \mathrm{j}$ et $\mathrm{t}=6040 \mathrm{j}$ ), des zones plastiques avec anti-écrouissage se développent dans les deux couches plus rigides qui constituent la partie supérieure du sol.

Les résultats du calcul sont représentés sur la figure 10 sous la même forme que pour les calculs précédents. On note sur cette figure que les déplacements horizontaux sous le pied du remblai se développent vers l'extérieur dans la partie inférieure du sol de fondation et vers l'intérieur dans sa partie supérieure 


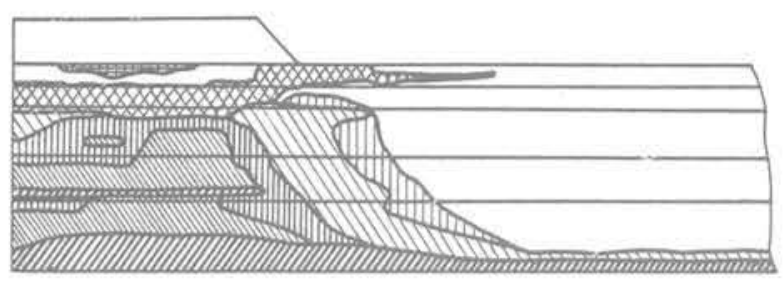

IIIII) zone plastifiée sous $\mathrm{H}_{r}=0,7 \mathrm{~m}$

atmin zone plostifibe sous $H_{r}=1,9 \mathrm{~m}$

andm zone plastifiéte sous $H_{r}=2,3 \mathrm{~m}$

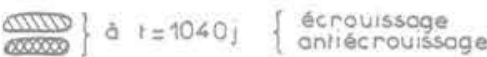

IIIII $\}$ à $l=6040 j \quad\left\{\begin{array}{l}\text { écrovissoge } \\ \text { antiéc rouissoge }\end{array}\right.$

Fig. 9

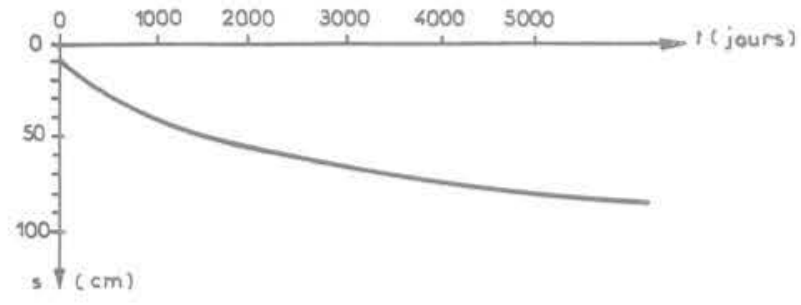

- Tassement sous l'axe du remblai

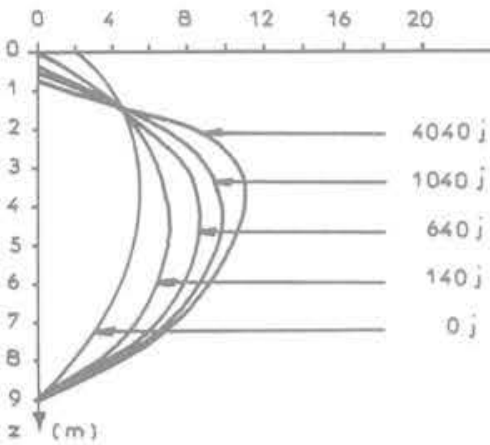

b. Déplocements horizonraux à la verticole du pied du remblai

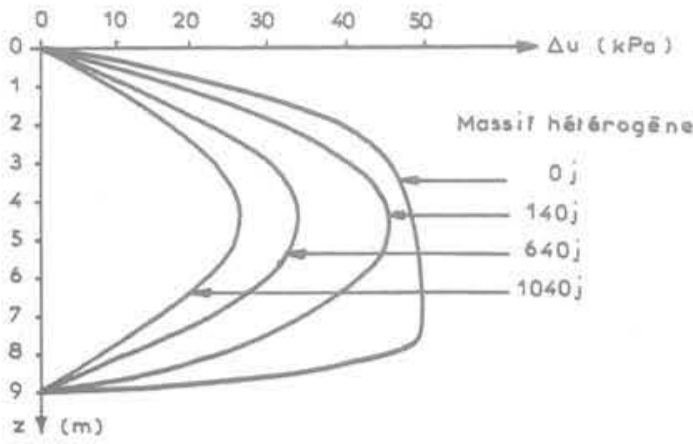

e- Surpressions interstirialles sous t'axe du remblai

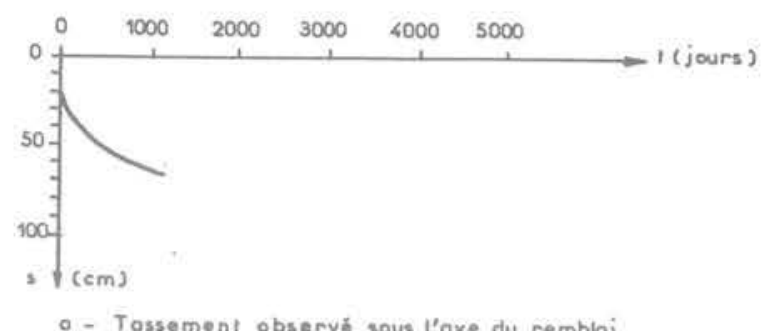

02468101214161820

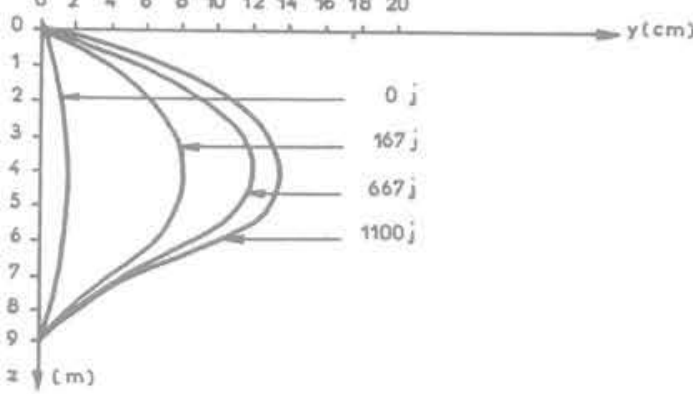

b- Dáplocemenls horizonloux observés a la verticole du pied du remblai

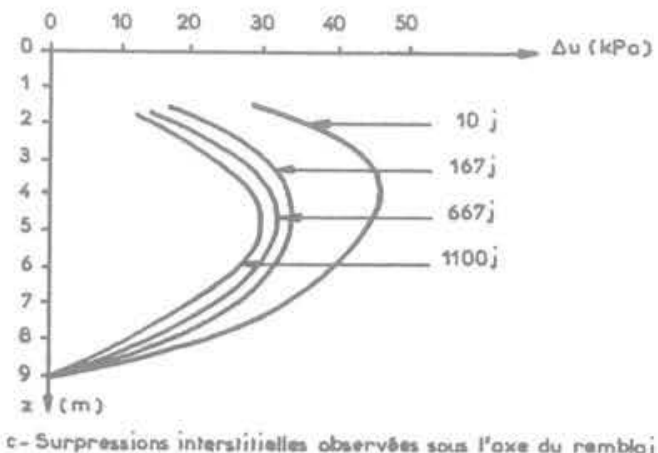

Fig. 11

plus rigide. D'autre part, la dissipation des surpressions interstitielles est plus rapide dans la partie supérieure de la couche.

\subsection{Comportement observé du remblai B de Cubzac-les-Ponts}

Pour faciliter la comparaison des résultats des mesures avec ceux des calculs, nous avons représenté sur la figure 11 les déplacements et surpressions interstitielles observés sous le remblai, sous une forme analogue à celle des figures 7,8 et 10 .

La partie supérieure des isochrones de surpression interstitielle n'a pas été représentée sur cette figure car le toit de la nappe oscille chaque année entre le niveau du terrain naturel et une profondeur de $1,5 \mathrm{~m}$.

\subsection{Comparaison des calculs et des mesures}

La figure 12 compare une sélection des courbes de tassement et de déplacements horizontaux et des isochrones de surpression interstitielle calculées avec les mesures correspondantes.

Pour les tassements sous l'axe du remblai, les mesures sont plus proches des calculs effectués en élasticité linéaire isotrope (sol homogène et sol hétérogène) et en élasticité linéaire anisotrope dans le cas du sol homogène. 


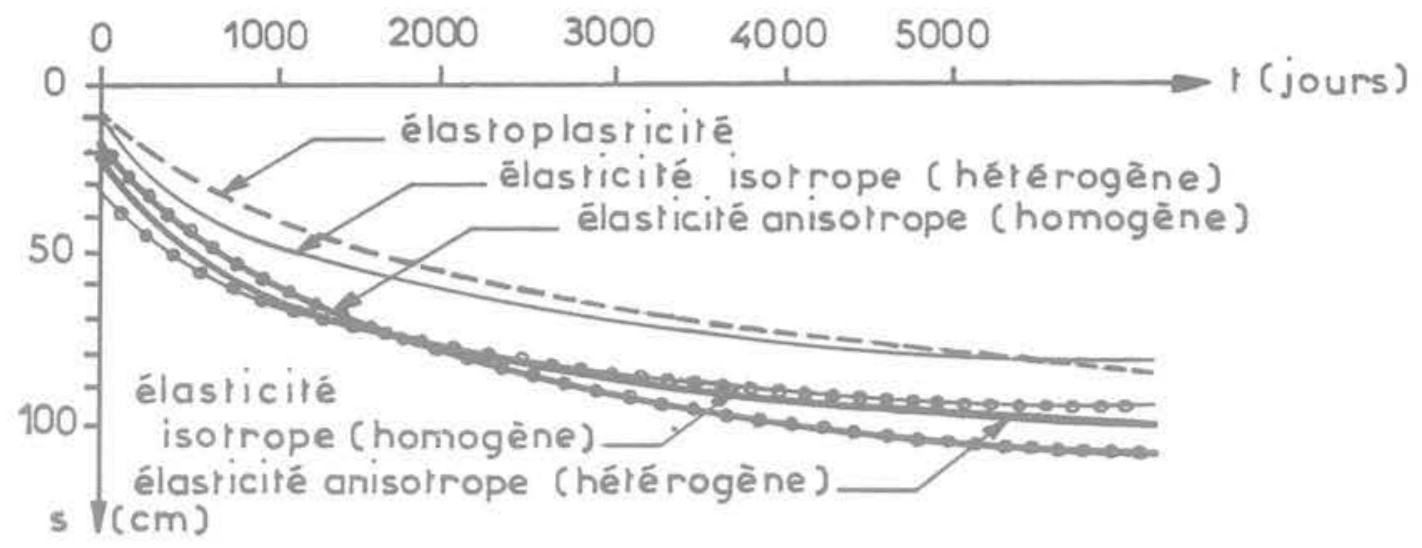

a - Tassemeni sous l'axe du remblai
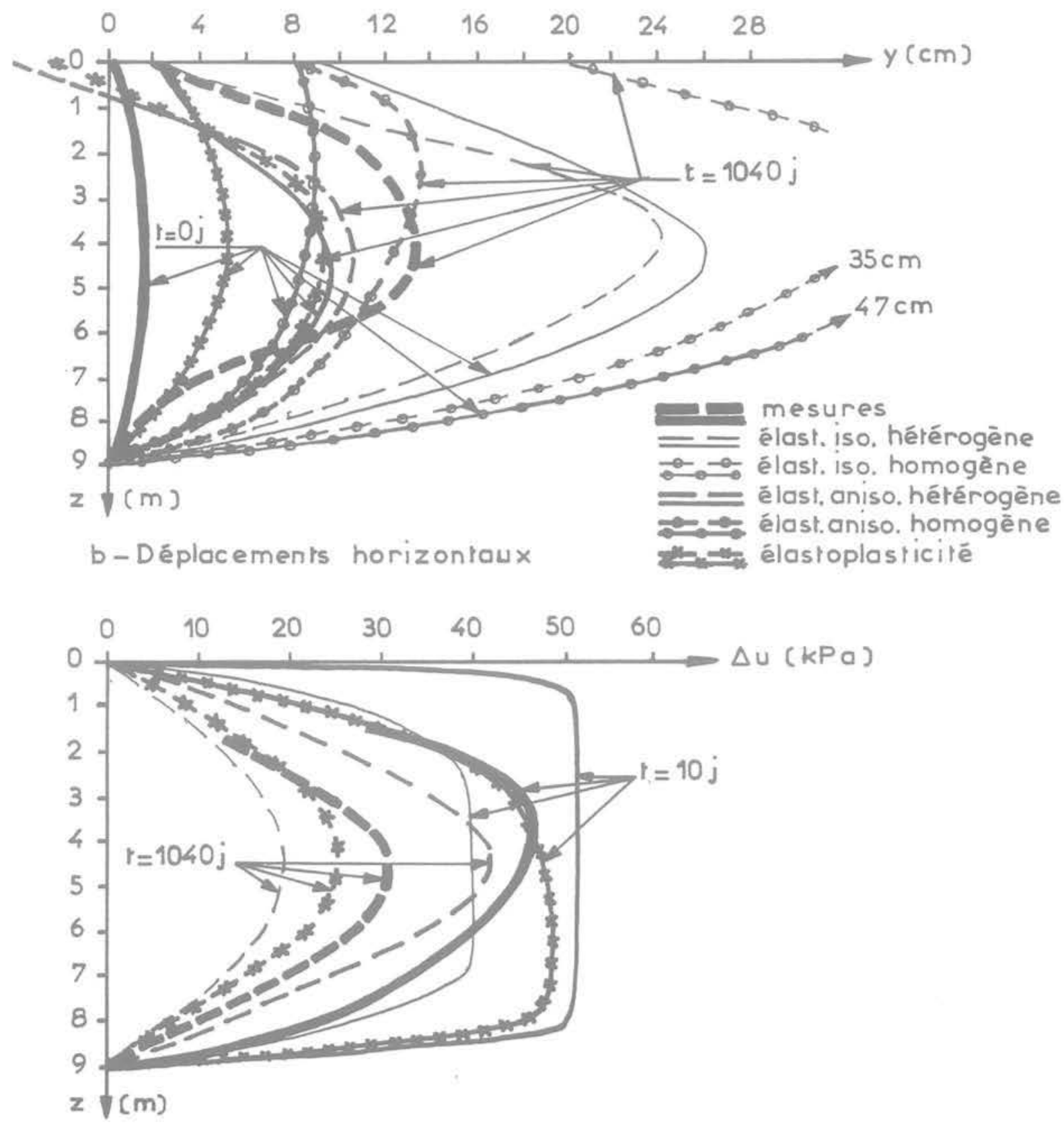

c - Surpressions interstirielles sous $1^{\prime \prime a x e}$ du remblai 
Du point de vue des déplacements horizontaux, la situation est toute différente: les deux calculs en élasticité linéaire isotrope sont à rejeter parce qu'ils prédisent des déplacements d'amplitude exagérée et dont l'évolution est en contradiction avec celle des mesures. Les déplacements immédiats des calculs élastiques anisotropes sont trop importants mais leur valeur à $\mathrm{t}=1040 \mathrm{j}$ est proche des mesures. Le calcul en élastoplasticité donne des résultats un peu meilleurs.

La comparaison des isochrones de surpression interstitielle montre que le calcul élastoplastique donne des résultats plus satisfaisants que les autres, notamment dans la moitié supérieure de la couche.

\subsection{Commentaires}

S'il existe dans chacune des parties de la figure 12 un ou plusieurs calculs en bon accord avec les mesures, aucune des hypothèses n'est vraiment satisfaisante vis-à-vis de l'ensemble des résultats analysés :

- l'élasticité linéaire isotrope conduit à des valeurs aberrantes des déplacements horizontaux,

- I'élasticité linéaire anisotrope est meilleure vis-à-vis des déplacements horizontaux mais moins bonne pour les tassements,

- I'élastoplasticité type Cam-clay modifié est la meilleure pour les déplacements horizontaux et les surpressions interstitielles, mais la pire pour le tassement sous l'axe du remblai.

Même si l'élastoplasticité semble avoir un léger avantage sur les autres modèles de calcul, il est évident que les recherches doivent se poursuivre si l'on veut obtenir un jour des prédictions numériques fiables des déplacements, contraintes et pressions interstitielles dans tout le sol de fondation.

On pourrait tenter d'améliorer les prévisions des calculs en jouant sur les valeurs des paramètres mécaniques du sol de fondation. A part l'élasticité isotrope, qui donne des résultats qualitativement inexacts, les modèles de calcul utilisés conduisent en effet à des erreurs quantitatives que l'on peut espérer corriger en modifiant la valeur des nombreux paramètres :

- 8 paramètres seulement dans le cas de l'élasticité anisotrope (sol homogène), mais

- 40 paramètres pour l'élasticité anisotrope dans le cas du sol hétérogène divisé en 5 couches et

- 55 paramètres dans le cas des calculs élastoplastiques avec écrouissage.

Dans le cadre de l'étude rapportée ici, on a refusé d'entrer dans le jeu arbitraire des études paramétriques. Ce choix s'explique par trois raisons :

- d'une part, on ne disposait pas de valeurs mesurées des paramètres qui puissent garder leurs modifications ultérieures à l'intérieur de limites raisonnables,

- d'autre part, le coût important des calculs élastoplastiques interdisait en pratique leur multiplication,

- et enfin, il a été jugé préférable de continuer de perfectionner le modèle de calcul dans le sens d'une élastoplasticité anisotrope avec écrouissage, plutôt que de rester au niveau du modèle Cam-clay modifié (ce travail est en cours au L.C.P.C.).

II peut être utile de faire un dernier commentaire sur les calculs présentés dans cet article: l'utilisation des méthodes numériques est rarement exempte de problèmes de convergence, d'oscillations des résul- tats, etc. qui font que les résultats publiés ont souvent été précédés de calculs préliminaires variés, destinés à améliorer le maillage ou à tester le choix des incréments de temps. Les calculs effectués dans le cadre de la présente étude ont comporté un certain nombre d'étapes préliminaires de ce genre, destinées à tester le maillage mais non les valeurs des paramètres mécaniques du sol.

\section{Conclusion}

La comparaison sur le cas du remblai B du site expérimental de Cubzac-les-Ponts des possibilités des modèles élastique isotrope, élastique orthotrope de révolution et élastoplastique isotrope avec écrouissage (Cam-clay modifié) pour l'analyse numérique des problèmes de consolidation des sols de fondation compressibles a mis en évidence les défauts qualitatifs de l'élasticité isotrope et les possibilités offertes par l'élasticité anisotrope et l'élastoplasticité. Pour la prédiction des déplacements horizontaux du sol de fondation sous les remblais, en particulier, le progrès réalisé par abandon de l'élasticité isotrope dans les calculs de consolidation est très sensible puisque l'on a pu obtenir pour la première fois des déplacements horizontaux évoluant vers l'extérieur au cours de la consolidation.

Toutefois, des études complémentaires, utilisant des valeurs des paramètres déterminées en laboratoire sur des échantillons de sol intact, seront encore nécessaires si l'on veut démontrer la validité de l'une ou l'autre de ces deux méthodes, qu'il n'est pour l'instant pas question d'utiliser au niveau de l'élaboration des projets.

\section{Remerciements}

La mise au point de la version du programme ROSALIE-Groupe 9 utilisée pour cette étude a été beaucoup facilitée par les conseils de MM. Franck, Guellec et Humbert, auxquels les auteurs expriment leur gratitude.

\section{Références bibliographiques}

Baghery S. (1980). Probabilités et statistiques en mécanique des sols: analyse probabiliste de la stabilité et des tassements de remblais sur sols compressibles (site expérimental de Cubzac-lesPonts). Thèse de docteur-ingénieur. École Nationale des Ponts et Chaussées, Paris, déc. 1980, 277 pages.

Belkeziz A., Magnan J. P. (1982). Analyse numérique de la consolidation bidimensionnelle des sols élastoplastiques. Traitement par la méthode des éléments finis et application au remblai expérimental B de Cubzac-lesPonts. Laboratoire central des Ponts et Chaussées. Rapport de Recherche à paraître.

Christian J.T., Boehmer J.W. (1970). Plane strain consolidation by finite elements. Proc. ASCE, Journal of S. M. F.D., vol. 96, n SM4, 1436-1457.

Christian J.T., Boehmer J.W., Martin P.P. (1972). Consolidation of a layer under a strip load. Proc. ASCE. Journal of S.M. F. D., vol. 98, n SM7, 693-707. 
Dang M.T., Magnan J.P. (1977). Application des modèles élastoplastiques de l'Université de Cambridge au calcul du comportement d'un remblai expérimental sur sols mous. Laboratoire Central des Ponts et Chaussées, Rapport de Recherche LPC $n^{\circ} 74$, 106 pages.

Garnier J. (1973). Tassements et contraintes. Influence de la rigidité de la fondation et de l'anisotropie du massif. Thèse de doctorat de spécialité. Université Scientifique et Médicale de Grenoble, 193 pages.

Guellec P., Ricard A., Humbert P. (1976). Notice de présentation du programme Rosalie. Laboratoire central des Ponts et Chaussées. Rapport du Département de Géotechnique.

Hwang C.T., Morgenstern N.R., Murray D.W. (1971). On solutions of plane strain consolidation problems by finite element method. Canadian Geotechnical Journal, vol. $8, n^{\circ} 1,109-117$.

Hwang C.T., Morgenstern N.R., Murray D.W. (1972). Application of the finite element method to consolidation problems. Proc. Ist Intern. Conf. on Numerical Methods in Geomechanics, Vicksburg, 739-760.

Magnan J.P. Mieussens C., Queyroi D. (1978). Comportement du remblai expérimental B à Cubzac-les-Ponts. Revue Française de Géotechnique, $n^{\circ} 5,23-36$.

Ricard A. (1975). Étude des massifs continus à. comportement non linéaire. Application aux problèmes de génie civil. Laboratoire Central des Ponts et Chaussées. Rapport de recherche LPC $n^{\circ} 44$.

Sandhu R.S. (1972). Finite element analysis of consolidation and creep. Proc. 1st Intern. Conf. on Numerical Methods in Geomechanics, Vicksburg, 697-733.
Sandhu R.S. (1976). Variational principles for finite element analysis of consolidation. Proc. 2nd Intern. Conf. on Numerical Methods in Geomechanics, Blacksburg, 20-41.

Sandhu R.S., Wilson E.L. (1969), Finite element analysis of seepage in elastic media. Proc. ASCE, Journal of Engineering Mechanics Div., vol. 95, $n^{\circ}$ EM3, 641-652.

Shahanguian S. (1980). Détermination expérimentale des courbes d'état limite de l'argile organique de Cubzac-les-Ponts. Thèse de docteur-ingénieur. Université Pierre et Marie Curie (Paris VI). Avril 1980.

Soulié M. (1978). Les éléments finis: outil de prédiction. Application aux remblais sur sols compressibles. Coopération France-Québec: E.N.P.C.Université Laval. Séminaire de formation continue sur les Nouvelles méthodes d'étude des remblais sur sols compressibles.

Yokoo Y., Yamagata K, Nagaoka H. (1971 a). Finite element analysis of consolidation following undrained deformation. Soils and Foundations, vol. 11, $n^{\circ} 4$, 37-57.

Yokoo Y., Yamagata K., Nagaoka H. (1971 b). Variational principles for consolidation. Soils and Foundations, vol. 11, $n^{\circ} 4,25-35$.

Yokoo Y., Yamagata K., Nagaoka H. (1971 c). Finite element method applied to Biot's consolidation theory. Soils and Foundations. Vol. 11, $n^{\circ} 1,29-45$. 
